\title{
Differential Impact of Acclimation and Acute Water Deprivation in the Expression of Key Transcription Factors in Soybean Roots
}

\author{
Fábia Guimarães-Dias $^{1}$ • Anna C. Neves-Borges ${ }^{2} \cdot$ Alessandra J. Conforte $^{1}$. \\ Leonardo Giovanella-Kampmann ${ }^{1}$. André V. J. Ferreira ${ }^{4}$ • Regina M. S. Amorim ${ }^{4}$. \\ Magda A. Benevent ${ }^{4}$ - Maria Eugênia Lisei de $\mathrm{Sá}^{4} \cdot$ Rosilene O. Mesquita ${ }^{6}$. \\ Fabiana A. Rodrigues ${ }^{5}$ - Alexandre L. Nepomuceno ${ }^{5}$ - Eduardo Romano ${ }^{4}$. \\ Marcelo E. Loureiro ${ }^{3}$. Maria Fátima Grossi-de-Sá ${ }^{4} \cdot$ Márcio Alves-Ferreira $^{1}$ (D)
}

Published online: 22 June 2016

(C) Springer Science+Business Media New York 2016

\begin{abstract}
Soybean (Glycine max) is one of the major world commodities. In order to increase the soybean yields, it has been searched drought-tolerant cultivars, once the drought is the major constraint to soybean grown. Therefore, it is crucial to elucidate the molecular mechanisms associated with drought tolerance. Here, the in silico approach allowed us to identify 12 genes belonging to six different transcription factor
\end{abstract}

Fábia Guimarães-Dias and Anna C. Neves-Borges contributed equally to this work.

Electronic supplementary material The online version of this article (doi:10.1007/s11105-016-0993-z) contains supplementary material, which is available to authorized users.

Márcio Alves-Ferreira

alvesfer@uol.com.br

Fábia Guimarães-Dias

fabiadg@gmail.com

Anna C. Neves-Borges

annacrisnb@yahoo.com.br

Alessandra J. Conforte

conforteaj@gmail.com

Leonardo Giovanella-Kampmann

leonardogiovanella@hotmail.com

André V. J. Ferreira

eng.andre.julio@gmail.com

Regina M. S. Amorim

rmsamorim@yahoo.com.br

Magda A. Benevent

mabeneventi@gmail.com

Maria Eugênia Lisei de Sá

eugenialisei@gmail.com families in soybean that have been associated with key events on drought response. The expression pattern of each gene was investigated by qPCR in root samples of drought-sensitive and drought-tolerant cultivars undergoing drought stress in potbased (PSys) and hydroponic (HSys) cultivation systems. GmaxMYC2-like 2 was induced under abrupt drought conditions in HSys in both cultivars, whereas GmaxAREB1-like 1
Rosilene O. Mesquita rosilenemesquita@gmail.com

Fabiana A. Rodrigues deganuti@gmail.com

Alexandre L. Nepomuceno nepo@cnpso.embrapa.br

Eduardo Romano romano@cenargen.embrapa.br

Marcelo E. Loureiro mehlers@mail.ufv.br

Maria Fátima Grossi-de-Sá fatima.grossi@embrapa.br

1 Department of Genetics, Instituto de Biologia, Universidade Federal do Rio de Janeiro (UFRJ), Av. Prof. Rodolpho Paulo Rocco, s/n, $2^{\circ}$ andar-sala A2-93, CSS, Rio de Janeiro, RJ 21941-901, Brazil

2 Department of Botany, Universidade Federal do Estado do Rio de Janeiro (UNIRIO), Rio de Janeiro, RJ 22290-240, Brazil 
and GmaxDREB2A-like were highly induced only in the PSys. However, GmaxMYB2-like 1, GmaxMYB2-like 2, GmaxRD26/NAC-like 1, GmaxRD26/NAC-like 2, GmaxAREB1-like 2, and GmaxDREB1A-like were upregulated in both systems, while the GmaxHB6-like and GmaxHB13like are repressed under all of the investigated conditions. Exogenous abscisic acid (ABA) treatment was used to identify those genes belonging to the ABA-dependent drought response. The genes identified in this work have potential application for the improvement of drought resistance in soybean and markers for breeding programs.

Keywords Drought adaptation · Glycine max · Marker assistance selection · Transcription factors $\cdot$ Water deficit

$\begin{array}{ll}\text { Abbreviations } & \\ \text { ABA } & \text { abscisic acid } \\ \text { ABAD } & \text { ABA dependent } \\ \text { ABAI } & \text { ABA independent } \\ \text { ABF } & \text { ABA-responsive transcriptional } \\ & \text { factors } \\ \text { ABRE/ABFs } & \text { ABA-responsive element } \\ & \text { binding/ABRE-binding protein } \\ \text { BG } & \text { background } \\ \text { BZIP } & \text { basic leucine zipper } \\ \text { DREB } & \text { dehydration-responsive element binding } \\ \text { HB } & \text { homeobox domain HD-zip } \\ \text { HSys } & \text { hydroponic system } \\ \text { MYB } & \text { myeloblastosis oncogene } \\ \text { MYC } & \text { myelocytomatosis oncogene } \\ \text { NAC } & \text { nitrogene assimilation control protein } \\ \text { PSys } & \text { pot-based system } \\ \text { BR 16 } & \text { sensitive cultivar } \\ \text { Embrapa } 48 & \text { tolerant cultivar } \\ \text { Iw } & \text { water potential }\end{array}$

\section{Introduction}

Soybean (Glycine max [L.] Merr) is a widely grown and commercialized legume because of its high oil (average $20 \%$ ),

Plant Biology Department, Universidade Federal de Viçosa (UFV), Viçosa, MG 36570-900, Brazil

4 Genetic Resources and Biotechnology (CENARGEN), Brasília, DF 70770-901, Brazil

5 Embrapa Soybean, Distrito de Warta, Londrina, PR 86001-970, Brazil

6 Department of Fitotecnia, Universidade Federal do Ceará (UFC), Ceará, CE 60020-181, Brazil protein (average $40 \%$ ), mineral, and macronutrient concentration and is used worldwide in the food and pharmaceutical industries, as well as in biodiesel production (Clemente and Cahoon 2009). Thus, this Asiatic species has been largely grown in both temperate and tropical regions (Comlekcioglu and Simsek 2011). However, among the legume species, soybean is considered the most sensitive plant to drought which reduces the yield by approximately $40 \%$ (Thao and Tran 2012). Drought is the most damaging abiotic stress, affecting every stage of plant growth and development and contributing drastically to soybean yield loss (Hussain et al. 2011; Le et al. 2011). For example, the phenotypic uniformity of soybean plant is affected if water deficit occurs during the germination and emergence phase (Thao and Tran 2012). Moreover, flower abortion if water deficit occurs during reproduction phase, while the seed weight is affected if drought occurs during grain filling (Tran and Mochida 2010). Therefore, the elucidation of plant drought stress response mechanisms is a major step for the evaluation, selection, and development of soybean drought-tolerant lines, which is important in reducing the risk of yield loss due to drought stress in drought-prone production areas (Osakabe et al. 2014).

The Brazilian soybean cultivars BR 16 and Embrapa 48 were previously characterized as drought sensitive and tolerant, respectively (Casagrande et al. 2001; Oya et al. 2004; Texeira et al. 2008). These reports showed that, among several cultivars, Embrapa 48 had higher rate germination, higher length of seedling, and higher dry biomass under moderate and severe drought stress when compared with other cultivars available. On the other hand, BR 16 cultivar is highly affected by water deficit showing the worse indicators (Oya et al. 2004). These results show that Embrapa 48 and BR 16 are excellent models for the study of drought tolerance in soybean (Guimarães-Dias et al. 2012; Ferreira Neto et al. 2013; Marcolino-Gomes et al. 2013).

The responses at the molecular level to water deficit can be evaluated by distinct experimental approaches. The most traditional experimental approach uses plants grown in pots with soil (pot-based systems (PSys)) combined with a specific routine of watering. This system attempts to reproduce field conditions but requires careful physiological monitoring (StolfMoreira et al. 2010b; de Paiva Rolla et al. 2014; Mayer Weber et al. 2014). Alternatively, water deficit can be mimicked using plants that are cultivated in hydroponic systems (HSys) combined with an abrupt water privation by removing the plant from the nutrition solution (Cowan 1965). This system, in which water privation occurs quickly, is a reliable option for studying the early responses to water deficit (Stolf-Moreira et al. 2011; Marcolino-Gomes et al. 2013). However, this sudden water deficit does not allow to access the molecular processes associated to plant acclimation (Munns et al. 2010). 
The drought stress response mechanisms involve molecular, cellular, and physiological changes that are triggered by a complex molecular signaling cascade and that allow some plants to naturally minimize or prevent the damaging effects of water privation (Fang and Xiong 2015; Mutava et al. 2015). This regulatory signaling network is governed by abscisic acid (ABA)-dependent and ABA-independent pathways (Shinozaki and Yamaguchi-Shinozaki 1996; Nakashima et al. 2014). In both of these pathways, transcriptional factors (TFs) have been identified, which are key regulators of drought stress response processes and include AtAREB $1 / A B F$, AtMYB, $A t M Y C$, and AtRD26/NAC of the ABA-dependent pathway, as well as AtDREB1A/CBF3, AtDREB2A, and AtHD-ZIPI of the ABA-independent pathway (Yoshida et al. 2014).

The overexpression of the TFs belonging to the classical ABA-dependent or ABA-independent pathways, such as nitrogene assimilation control (NAC), basic leucine zipper (Bzip), myeloblastosis oncogene (MYB), myelocytomatosis oncogene (MYC), and dehydration-responsive element binding (DREB), promotes tolerance to drought stress in different species, including Arabidopsis, rice, and soybean (Le et al. 2011; Xu et al. 2011; Pandey et al. 2014; Miyamoto et al. 2015)

In this study, we used an in silico approach to identify the soybean homologs of the Arabidopsis transcriptional factors responsive to drought (TFRDs) that are involved in the ABA dependent (AREB, MYB, MYC, and RD26/NAC) and ABA independent (DREB1A, DREB2A, HB6, and HB13) in response to drought. The expression analysis of these transcriptional factors was performed in both experimental approaches (HSys and PSys) using roots of sensitive and tolerant cultivar, since most of these soybean genes are more expressed in the roots compared with leaves (Schmutz et al. 2010).

\section{Results}

\section{In Silico Identification and Characterization of the Soybean Transcriptional Factors That Are Responsive to Drought}

With the aim of identifying and characterizing the TFRD homologs to Arabidopsis thaliana in soybean, we first selected A. thaliana drought-responsive genes using information from the literature (Yamaguchi-Shinozaki and Shinozaki 2006; Shinozaki and Yamaguchi-Shinozaki 2007). Then, the expression pattern of the selected TFs (AtAREB1 (bZIP), AtDREB1A, AtDREB2A, AtHD-ZIP, AtMYB2, AtMYC2, and $A t R D 26 / N A C$ ) was double-checked using the Genevestigator software (Hruz et al. 2008).

Digital expression analyses confirmed previous results for the selected Arabidopsis TFRDs belonging to the ABAdependent pathway, demonstrating that these genes are induced by ABA stimulus and water deficit. Among the TFRDs belonging to the ABA-independent pathway, only $A t H B 13$ showed an unexpected expression pattern according to Genevestigator. The expression of $A t H B 13$ is downregulated under ABA stimulus and drought stress conditions (Fig. S1).

For each selected Arabidopsis TFRD, the putative homo$\log$ of the Glycine max and Oryza sativa genomes was identified through a BLASTP search in the Phytozome database, followed by a neighbor-joining analysis and the construction of dendrograms. The identification of the $G$. $\max$ and $O$. sativa putative homologs of each species is described in the Material and Methods section. When the dendrogram analysis did not establish the putative soybean homolog gene, the soybean gene with the smallest $e$ value in BLASTP analysis was considered the putative homolog. This strategy allowed us to identify the 12 putative soybean genes that were homologs to Arabidopsis drought-responsive genes (Fig. 1). These genes were evaluated in Soybean eFP Browser (http://bar.utoronto.ca/efpsoybean/cgi-bin/efpWeb.cgi), and 7 out of the 12 have higher expression in roots than leaves (data not shown). Roots sense the edaphic water deficit, direct chemical signals to the shoots, and preservation of root growth despite that reduced water accessibility can contribute to drought tolerance through water foraging (Lynch 1995). Due to the expression pattern of the TFRD genes by eFP Browser and the importance of roots in drought tolerance, we decided to focus our gene expression analysis on soybean roots.

\section{Expression Profile Analysis of TFRDs Under Water Privation Conditions in PSys or HSys}

The expression profile of soybean TFRDs was investigated by qPCR on RNA samples from the roots of the sensitive (BR 16) and the tolerant (Embrapa 48) soybean cultivars. The GmaxRD26/NAC-like 1 and GmaxRD26/NAC-like 2 genes, putative homologs of AtRD26/NAC that belong to the ABAdependent pathway, showed fairly similar expression dynamics in HSys (Fig. 2a, b). The GmaxRD26/NAC-like 1 and GmaxRD26/NAC-like 2 genes were significantly upregulated in the samples of the both cultivars when subjected to water deficit in HSys. However, GmaxRD26/NAC-like 2 showed a higher induction in the sensitive cultivar (100 and $150 \mathrm{~min}$ ) (Fig. 2b). GmaxRD26/NAC-like 2 was weakly but significantly upregulated in both of the cultivars in PSys, while GmaxRD26/NAC-like 1 was upregulated only in the sensitive cultivar (Fig. 2a, b).

GmaxMYB2-like 1 and GmaxMYB2-like 2, putative homologs of AtMYB2 belonging to the ABA-dependent pathway, showed distinct expression profiles in the PSys. These genes were significantly induced in both of the cultivars under all of the evaluated condition, but GmaxMYB2-like 1 was more 


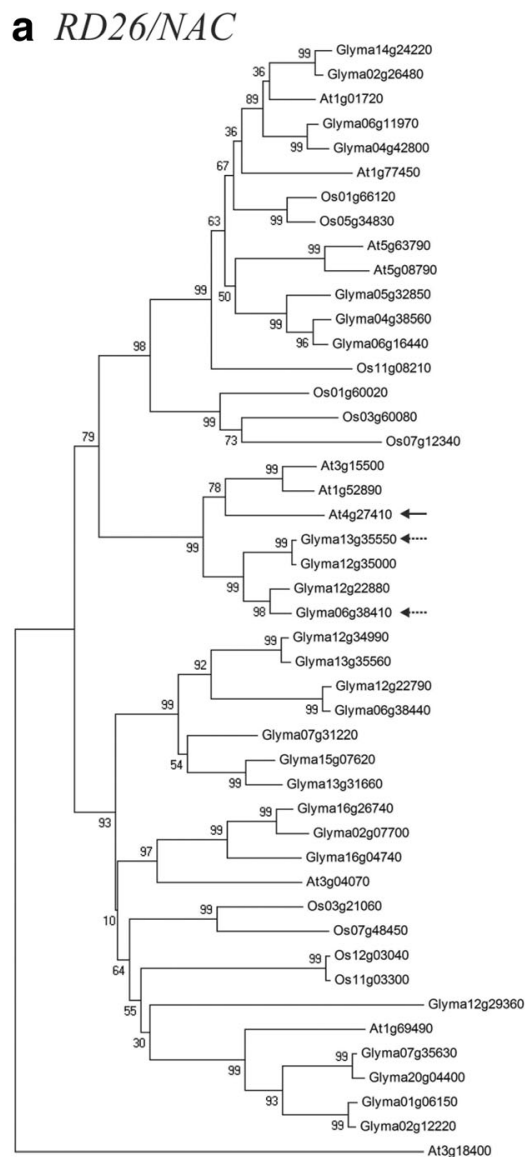

$\longmapsto$ b $M Y B 2$

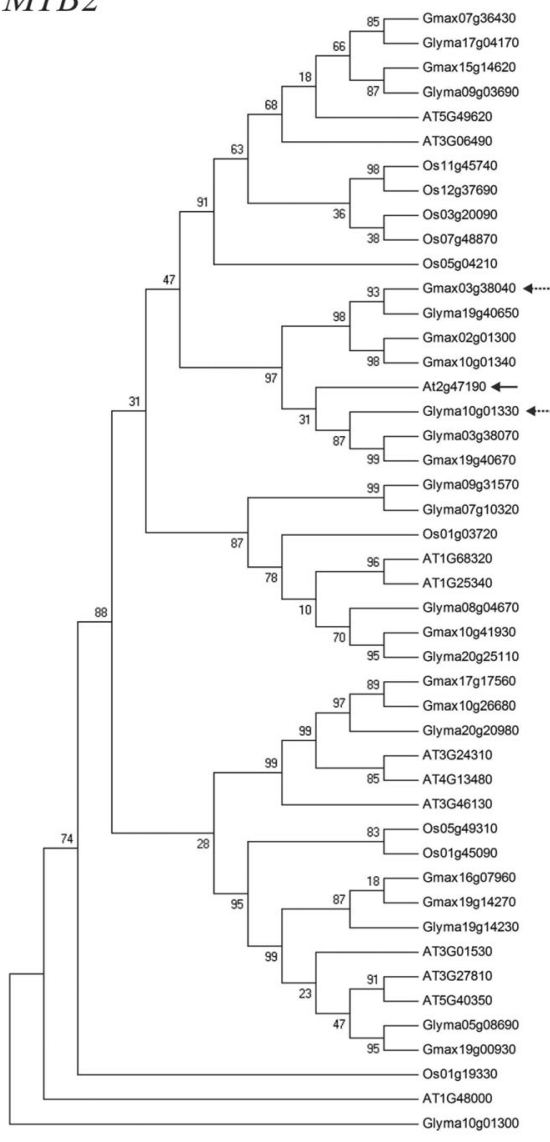

c $M Y C 2$

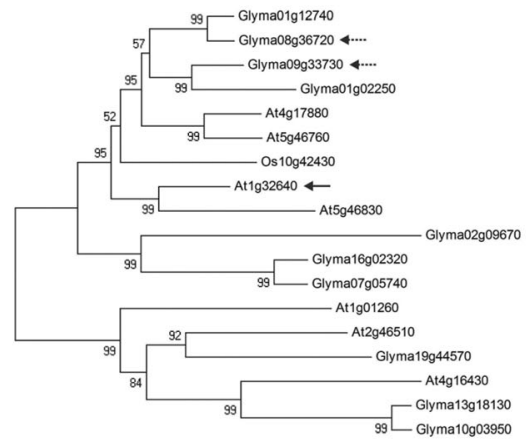

$\longmapsto$

d $A R E B I$

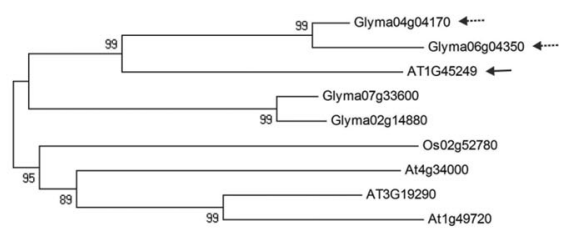

0.05 e $D R E B I A$

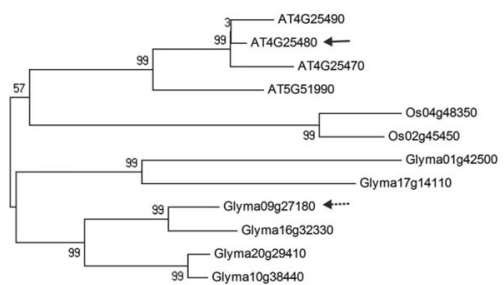

$\longmapsto$ g $H B 6$

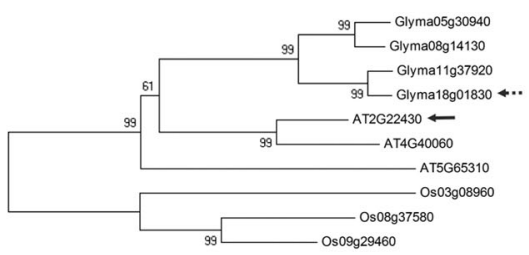

$\longmapsto 01$ h $H B 13$

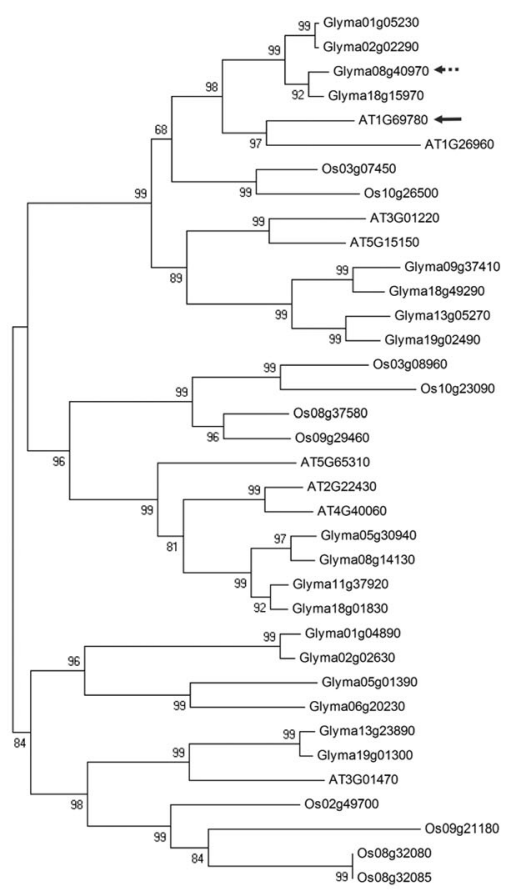

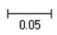


4 Fig. 1 Dendrograms of the Arabidopsis thaliana, Glycine max and Oryza sativa (outgroup) TFRDs, based on the amino acid sequences. a NAC/RD26 family, b MYB2 family, c MYC2 family, d AREB1/ABF2, e DREB1A/CBF3 family, f DREB2A family, g HB6 family, and h HB13 family. The multiple alignment was made using Clustal W2, and the dendrograms were built using the MEGA 4.0 software using the neighbor-joining and pair-wise deletion options with a consensus of 1000 bootstrap replicates. The arrows indicate the Arabidopsis reference key genes, and the dotted arrows indicate the respective homolog soybean genes that were selected for qPCR expression validation

induced in the sensitive cultivar in HSys (Fig. 2c). In contrast, GmaxMYB2-like 2 showed a similar induction in both of the cultivars in HSys but exhibited a contrasting expression pattern in PSys, being downregulated in the sensitive cultivar and upregulated in the tolerant cultivars (Fig. 2d).

The GmaxMYC2-like 1 and GmaxMYC2-like 2 genes, putative homologs of the Arabidopsis MYC2 gene belonging to the ABA-dependent pathway, also showed dissimilar expression profiles during the different drought stress conditions. GmaxMYC2-like 1 was upregulated in the tolerant cultivar under PSys and in both cultivars at the early time (T50 minutes) under HSys. In contrast, GmaxMYC2-like 1 was downregulated in the sensitive cultivar in PSys and at T100 minutes of HSys (Fig. 2e). GmaxMYC2-like 2 was upregulated during water deprivation in the sensitive and tolerant cultivar, while it is downregulated in both cultivars in PSys (Fig. 2f).

The GmaxAREB1-like 1 and GmaxAREB1-like 2 genes, the putative homologs of the Arabidopsis AREB1/ABF2 genes belonging to the ABA-dependent pathway, have a strikingly similar expression profile under PSys, in which both of these genes are significantly induced in both of the cultivars (Fig. 2g, h). In contrast, under HSys, GmaxAREB1-like 1 showed no significant difference during the time in which it was exposed to stress compared to the control sample (Fig. 2g), while GmaxAREB1-like 2 was significantly induced in the sensitive cultivar (T50 and T150 minutes) despite showing low expression levels. On the other hand, this gene was downregulated within $100 \mathrm{~min}$ in the tolerant cultivar (Fig. 2h).

GmaxDREB1A-like and GmaxDREB2A-like, homologs of Arabidopsis DREB1A and DREB2A belonging to the ABAindependent pathway, showed dissimilar dynamics of expression under most of the analyzed conditions (Fig. 2i, j). The GmaxDREB1A-like gene was significantly upregulated in tolerant soybean cultivars under PSys and HSys but was downregulated in the sensitive cultivar in T100 and T150 minutes. In contrast, GmaxDREB2A-like was significantly induced only in the tolerant cultivar under PSys, while this gene was downregulated under HSys in both of the cultivars (Fig. 2j).

The GmaxHB6-like and GmaxHB13-like genes also have Arabidopsis homologs that are responsive to drought through an ABA-dependent or ABA-independent pathway.
GmaxHB6-like and GmaxHB13-like were downregulated in the both of the cultivars under most of the conditions of PSys and HSys (Fig. 2k, 1).

In order to confirm the participation of the selected transcription factors in the ABA-dependent pathway, we evaluated the response of these genes to exogenous ABA application in the soybean leaves of both of the cultivars under PSys.

\section{Expression of the TFRDs in Response to ABA Stimulus}

We evaluate the TFRD differential expression in leaves of the sensitive and tolerant cultivars by qPCR, after ABA stimulus. Among the soybean putative TFRDs belonging to the ABAdependent pathway that were identified in our analysis, the expression of every gene, except for GmaxMYC2-like 2 and GmaxAREB1-like 1 (Fig. 3f, g), was upregulated by ABA stimulus (Fig. 3a, b, c, d, e, h). Among the evaluated genes, the GmaxRD26/NAC-like 1 and GmaxRD26/NAC-like 2 genes presented the highest levels of induction. As expected, all of the putative homologs of Arabidopsis genes belonging to the ABA-independent pathway (GmaxDREB1A-like, GmaxDREB2A-like, GmaxHB6-like, and GmaxHB13-like) were not sensitive to exogenous ABA stimulus (Fig. 3i, j, k, l).

\section{Promoter Analysis}

We used the POBO program (Kankainen and Holm 2004) to evaluate the enrichment of ten Cis elements that were related to drought, ABA, or osmotic stress, including the ABRE, MYB, and MYC Cis-regulatory elements (Tables S2 and S3) that we found in the promoter of the TF genes. We used the PLACE program (Higo et al. 1999) and literature data to identify these Cis elements (Harb et al. 2010; Umezawa et al. 2010). The statistical significance of their enrichment in the promoters in the ABA-dependent group (ABAD) and the ABA-independent group (ABAI) was evaluated as well, comparing the frequency of these motifs and the statistical significance of their enrichment in the promoters of each group and in the background set (comprising all of the promoter regions of the G. max genome-background (BG) model). This analysis revealed that one ABA-responsive element-binding motif (ACGTG) was enriched approximately seven times in the ABAD group compared to the ABAI group and two times compared to the BG group. Furthermore, another four ABAresponsive elements (ACGTGKC, RYACGTGGYR, CCACGTGG, and YACGTGGC) were enriched 4.5 to 12 times in the promoter mean of the ABAD group compared to the average $\mathrm{BG}$ promoters. These Cis-regulatory elements were not found in the promoter of the ABAI group (Table S2). In contrast, MYB-binding motifs (CNGTTR) were enriched approximately 1.5 times in the average promoters in the ABAD group compared to the ABAI group and enriched once compared to the BG group. In addition, two MYC-binding 

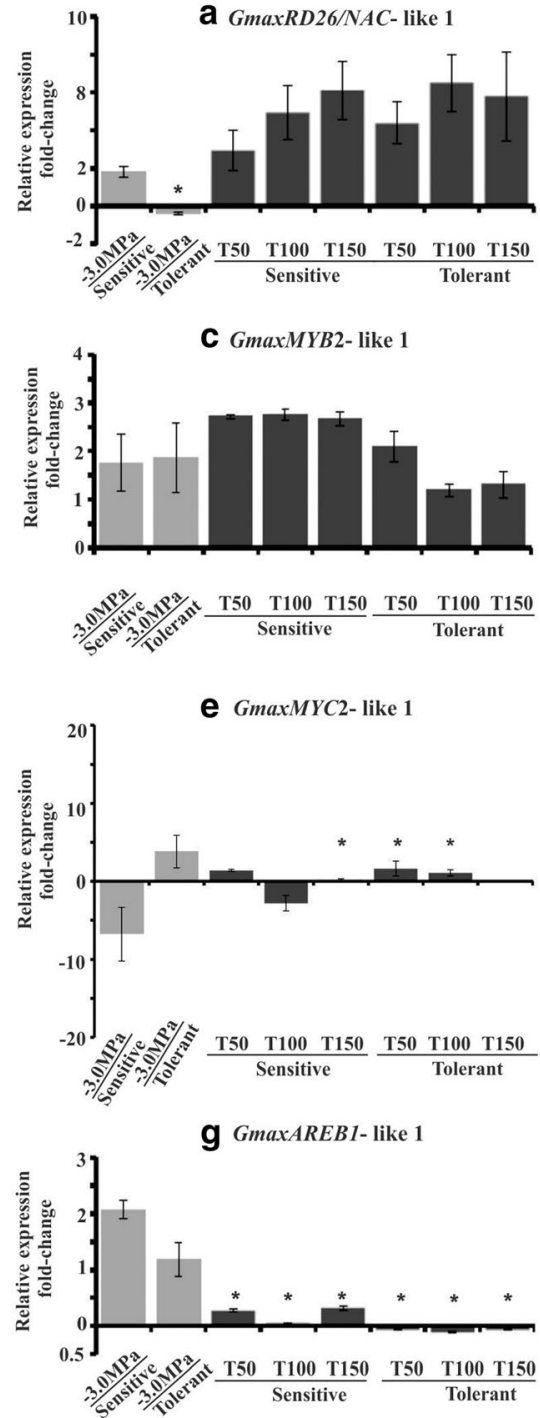

I GmaxDREB1A-like

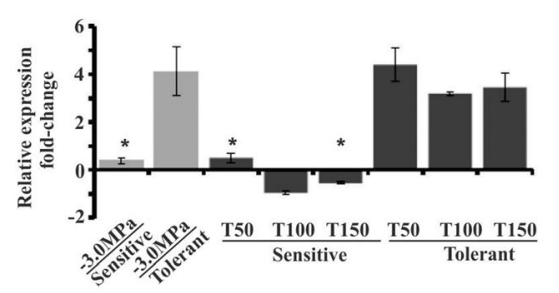

K GmaxHB6-like

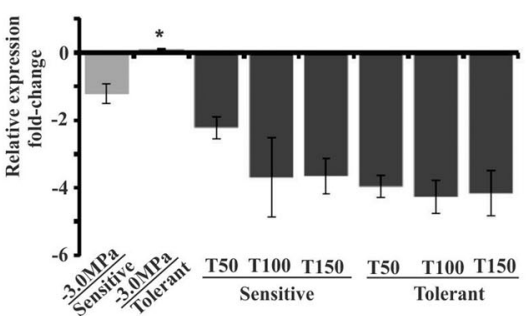

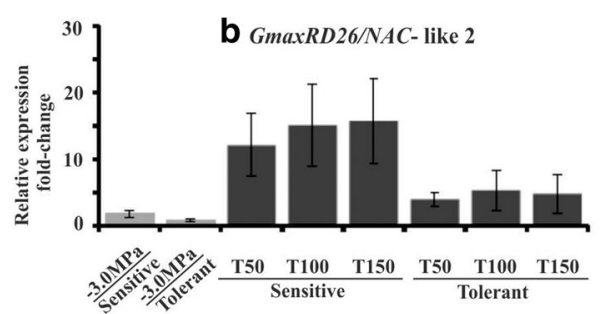
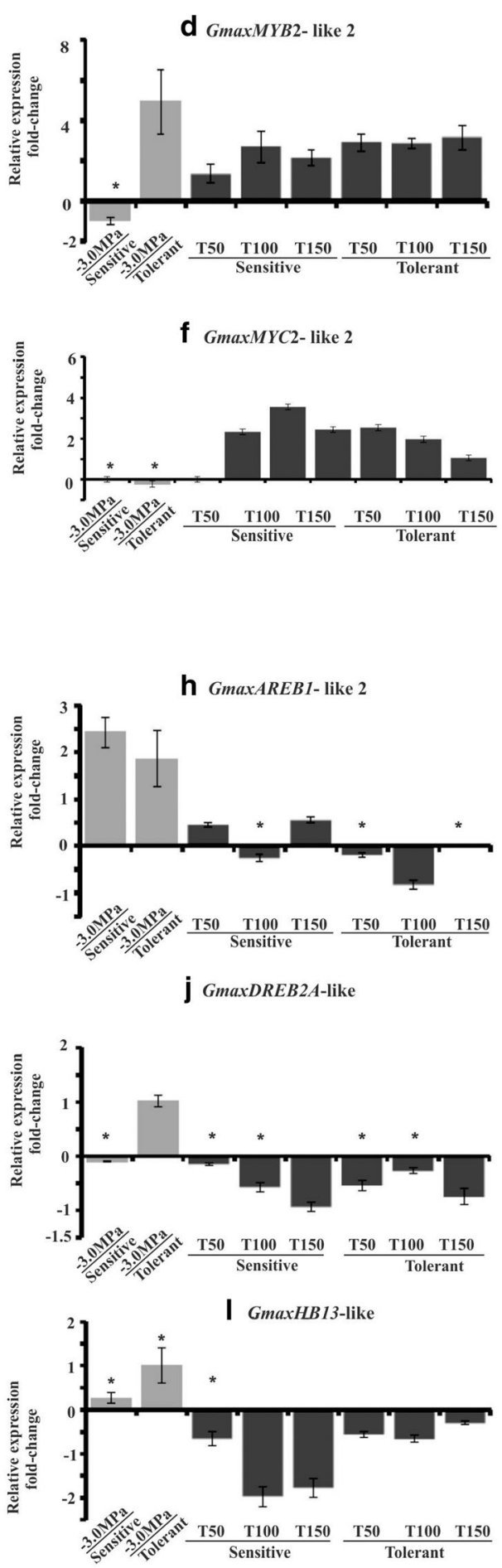

Psys $\square$ HSys 
4 Fig. 2 Fold change relative to the control from the soybean TFRDs in the roots of sensitive (BR 16) and tolerant (Embrapa 48) soybean genotypes during drought stress under PSys and HSys. a GmaxRD26/NAC-like 1, b GmaxRD26/NAC-like 2, c GmaxMYB2-like 1, d GmaxMYB2-like 2, e GmaxMYC2-like 1, f GmaxMYC2-like 2, g GmaxAREB1-like 1, h GmaxAREB1-like 2, i GmaxDREB1A-like, j GmaxDREB2A-like, k GmaxHB6-like, and I GmaxHB13-like. Transcription factor genes that are responsive to soybean genotypes are differentially regulated in the roots during drought stress conditions as established in $\left(\Psi_{\mathrm{W}}=-3.0 \mathrm{MPa}\right)$ or in HSys at $50 \mathrm{~min}(\mathrm{~T} 50), 100 \mathrm{~min}(\mathrm{~T} 100)$, and $150 \mathrm{~min}$ (T150) after water privation. Asterisks indicate the cases in which there were no significant differences between the samples under water stress and the control samples. The reference genes Fbox and UKN2 were used to normalize the qPCR data. The relative expression level (log 2 fold change) was calculated using the relative expression software tool (RESTC), and a subsequent statistical test of the analyzed CP values by a Pair-Wise Fixed Reallocation Randomization Test was performed

motifs (CACATG and CATGTG) were enriched approximately 1.8 times in the average promoters in the ABAI group compared to the $\mathrm{BG}$ and $\mathrm{ABAD}$ groups (Table $\mathrm{S} 3$ ).

\section{Discussion}

Herein, we identified and characterized the expression of 23 G. max TFRD genes that were putative homologs of genes that were previously characterized in Arabidopsis as key genes in the response to water deficit. We observed that the RD26/NAC, MYB, MYC, and HB13 families have approximately twice as many members in the soybean genome compared to the Arabidopsis genome (Fig. 1). This result is consistent with previous studies that showed that the soybean genome is palaeopolyploid and contains an abundance of homolog regions that are derived from the genome duplication. Nearly $75 \%$ of the genes in the soybean genome are multiple copies (Doyle and Egan 2010; Schmutz et al. 2010). Thus, the dendrogram analysis was crucial in matching the putative soybean TFRDs with the previously characterized homolog genes of Arabidopsis (Fig. 1).

After the in silico analysis, a careful study of the gene expression was conducted using $\mathrm{qPCR}$. The qPCR assays was performed on root samples since this organ is the first point of perception of drought stress by means of direct contact with soil (Vadez 2014). Roots are a very good model to study molecular mechanism important to drought early responses and acclimation in plants. Moreover, Fan et al. (2013) showed by RNAseq analysis that the total number of regulated genes in soybean roots is close to two times higher when compared to leaves during drought stress; 2956 genes were found regulated in roots and 1620 in leaves. As mentioned before, this is probably because the root is the first organ directly disturbed by drought stress.

The qPCR results showed significant differences in the expression profile of the selected genes, in the sensitive (BR
16) and tolerant (Embrapa 48) cultivars, when cultivated under two different water deprivation systems: (i) PSys, in which the water loss is slower, allowing the plant to acclimate to the unfavorable environmental condition, and (ii) HSys, in which the water loss is fast, not allowing the plant to acclimate to the stress condition.

We observed that GmaxAREB1-like 1, GmaxAREB1-like 2, GmaxMYB2-like 2, GmaxMYC2-like 1, and GmaxDREB2Alike genes are highly upregulated in PSys. In Arabidopsis, the AREB1/ABF gene controls the expression of the AtRD20 gene, while the $M Y B 2$ and $M Y C 2$ genes function as transcriptional activators in abscisic acid signaling and also activate directly the expression of AtRD22 gene (Abe et al. 2003; Wang et al. 2012). Besides, DREB2A controls the expression of AtRD29A (Narusaka et al. 2003). Our group previously showed that the GmaxRD20A-like and GmaxRD22-like expressions also were higher in plants grown under PSys (Neves-Borges et al. 2012). AtRD20A encodes for a caleosin protein that acts in the mobilization of lipids during seed germination, and it is implicated in drought tolerance by regulation of stomatal opening, plant growth, and water use efficiency (Aubert et al. 2011). AtRD22 encodes a member of the BURP protein family, and it is upregulated by salinity stress, exogenously supplied ABA, and dehydration drought (Yamaguchi-Shinozaki and Shinozaki 1993).

GmaxMYB2-like 1 and GmaxMYC2-like 2 genes are highly inducible in the HSys. Recently, it has been shown that WRKY1 is able to bind to the W-box domain in the promoter of MYB2 and thus to control its transcription in response to drought stress or ABA treatment (Qiao et al. 2016). MYC2 bHLH transcription factor is a transcriptional activator that acts as a master regulator to integrate signals from several pathways to coordinate plant stress response and development (Kazan and Manners 2013). It has suggested that MYC2 acts as a principal switch regulating both positive and negative interplay between $\mathrm{ABA}$ and JA signaling (Lorenzo et al. 2004; Bu et al. 2008; Kazan and Manners 2013). The GmaxRD26/NAC-like 1 and GmaxRD26/NAC-like 2 expressions have also a higher expression level in HSys when compared to PSys. The AtRD26/NAC gene is an important expression regulator of the $G L Y$ gene that encodes a protein of the glyoxalase I family. It is involved with oxidative stress tolerance modulating different plant physiology processes, such as limitation of the increase of ROS through their detoxification and photosynthesis (Fujita et al. 2004; Hoque et al. 2016). Indeed, Ferreira-Neto et al. (2013) showed that the NAC3 (soybean gene model Glyma06g38410; GmaxRD26/NAClike 1) have similar expression profile in tolerant and sensitive cultivar, under 50 and 100 min of stress in HSys. This result was similar with our results in the same conditions. However, this gene presents contrasting responses when, in PSys, evaluated in the two cultivars, been upregulated in BR 16 (sensitive cultivar) but downregulated in Embrapa 48 (tolerant 
a GmaxRD26/NAC-like1

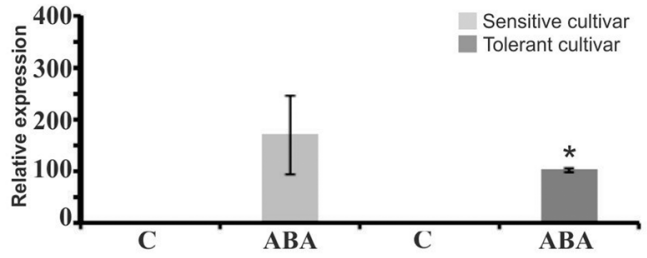

C GmaxMYB2-like1

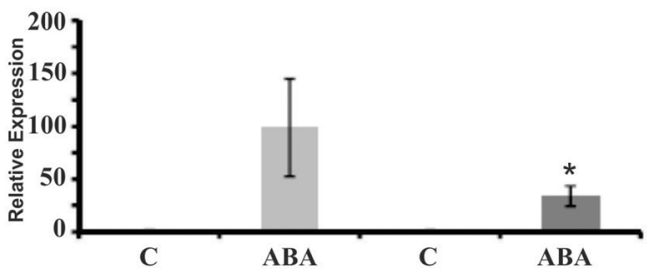

e GmaxMYC2-like1

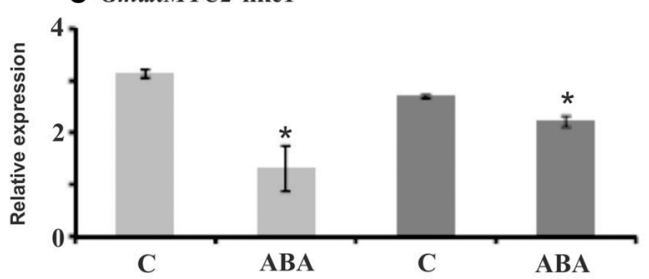

g GmaxAREB1-like1

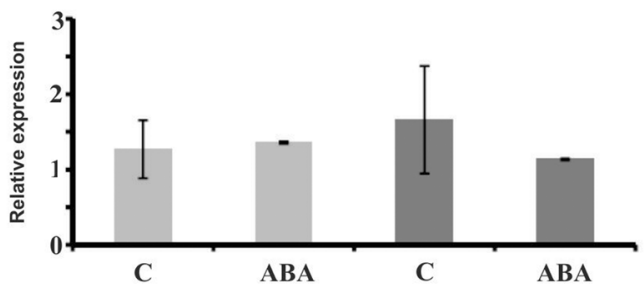

i GmaxDREB1A-like

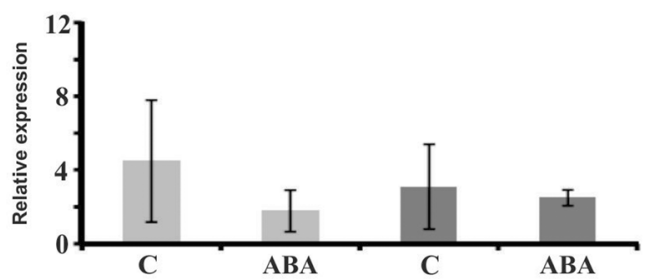

k GmaxHB6-like

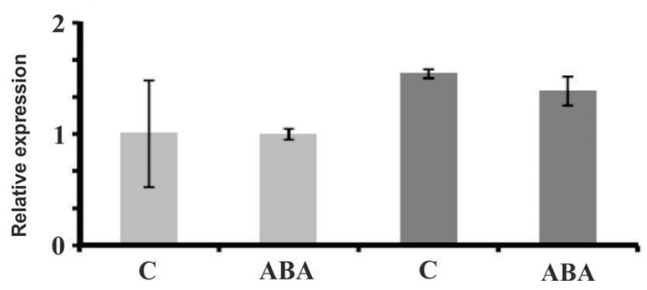

b GmaxRD26/NAC-like2

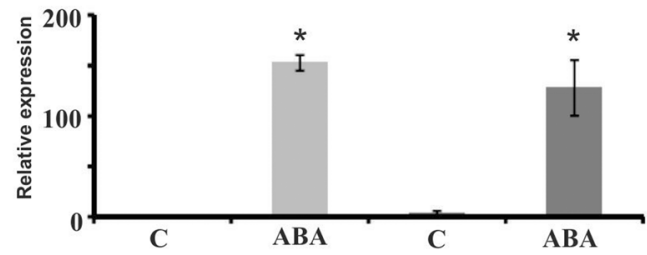

d GmaxMYB2-like2
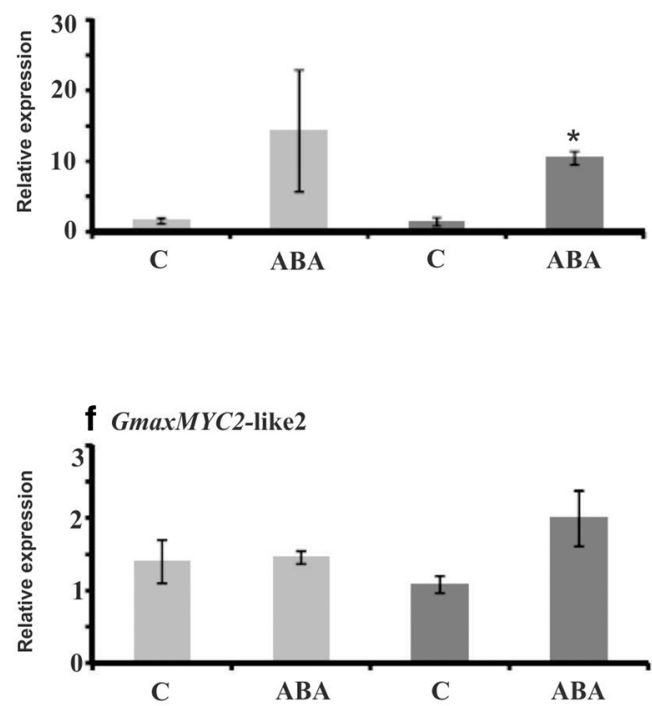

h GmaxAREB1-like2

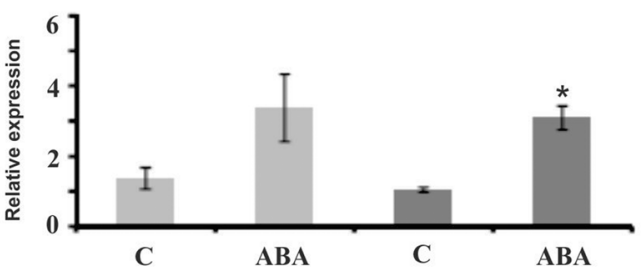

I GmaxDREB2A-like
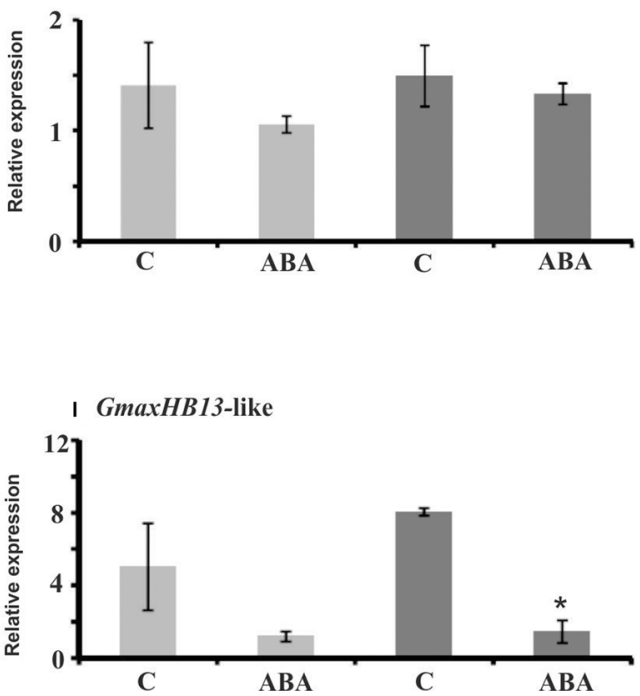
4Fig. 3 Expression profile analyses from the soybean TFRDs in the leaves of drought-sensitive and drought-tolerant genotypes under exogenous ABA stimulus. a GmaxRD26/NAC-like 1, b GmaxRD26/ NAC-like 2, c GmaxMYB2-like 1, d GmaxMYB2-like 2, e GmaxMYC2like 1,f GmaxMYC2-like 2, g GmaxAREB1-like 1, h GmaxAREB1-like 2, i GmaxDREB1A-like, j GmaxDREB2A-like, k GmaxHB6-like, and $\mathbf{I}$ GmaxHB13-like. The TFRDs from soybean genotypes were differentially regulated in leaves by exogenous ABA stimulus. The gray and black indicate the sensitive and tolerant cultivars, respectively. Control not stimulated $(C)$ or after 300 ppm of exogenous ABA $(A B A)$. The relative expression values, represented in the $Y$-axis, were obtained by $\mathrm{qPCR}$ experiments and calculated using the $2^{-\Delta \Delta \mathrm{Ct}}$ method, and ACT11 and Fbox were used as endogenous controls to normalize data. Asterisks indicate significant differences of ABA samples and control samples. (Student's $t$ test $\left.{ }^{*} p<0.05\right)$. Values are means $\pm \operatorname{SD}(n=2)$

cultivar; Fig. 2a). These results illustrate the importance of employing both systems of water deprivation (PSys and HSys) in order to better characterize gene expression during water deficit.

According to previous reports, the regulation of chromatin can modify the expression pattern of genes upregulated late under unfavorable environmental conditions (Sahu et al. 2013; Kim et al. 2015); therefore, the epigenetic memory might be manifested (Ding et al. 2013; Avramova 2015). This mechanism that occurs during the subsequent exposure of plants providing different responses from those during their first contact with stress may lead to the adaptation of the plant to new stress conditions (Fujimoto et al. 2012; Kilian et al. 2012). Ding et al. (2012) showed that AtMYB2, AtMYC2, $A t A R E B 1$, and AtDREB1A have modulated expression after one cycle of drought stress, suggesting that these genes are targets of epigenetic modulation during water deficit, while AtDREB2A is not modulated.

In addition to new insight into the control of gene expression during drought stress in soybean, our work is useful for the identification of useful molecular markers for soybean marker-assisted breeding. Recent data have identified and characterized the expression of the $L E A-D 11$ gene in four drought-tolerant cultivars, two drought-moderate cultivars, and one drought-sensitive cultivar (Savitri et al. 2013). The $L E A-D 11$ gene can be used as a molecular marker and is able to differentiate between drought-susceptible, drought-moderate, or drought-tolerant cultivars. We observed contrasting expression patterns of the TFRDs between tolerant (Embrapa 48) and sensitive (BR 16) cultivars in both of the drought stress systems. For instance, the induction level of the GmaxMYB2-like 2, GmaxMYC2-like 1, GmaxDREB1A-like, and GmaxDREB2A-like genes in PSys was higher in the drought-tolerant cultivar. This expression profile suggests that these genes may be good candidates for maker-assisted breeding in soybean.

In conclusion, most of the genes evaluated in the work show striking differences in the level of expression when distinct cultivation systems were tested. Differences in the expression were also observed when distinct cultivars are tested, highlighting the importance of careful evaluation of the expression to score drought response. We suggest that HSys may be the best platform to apply cycles of water deficit and, therefore, to study epigenetic memory in soybean. However, further research is needed to validate this method as bona fide to investigate epigenetic mechanism during drought events.

Our results suggest a high level of conservation between soybean and Arabidopsis in the expression profile of TFRDs in response to ABA. We also demonstrated that the MYB Cisregulatory element and $\mathrm{ABA}$ response elements are significantly enriched in promoters of the ABA-dependent group compared to the ABA-independent and/or background groups (BG) (Fig. 3a, b, c, d, f, g, h). This result suggests that MYB and ABRE motif recruitment might be an important modulator of gene expression in the ABA-dependent pathway. These data are consistent with previous promoter analyses of ABAinducible genes that identified the ABA-responsive element (ABRE PyACGTGG/TC) (Busk and Pages 1998). On the other hand, since most of the effects described in response ABA occur in leaves, after ABA stimulus, we addressed the gene expression analysis to this organ. Our results showed that only the genes of the ABA-dependent group, except for GmaxMYC2-like 2 and GmaxAREB1-like 1, were upregulated by ABA stimulus.

\section{Material and Methods}

\section{Identification In Silico of Transcriptional Factors That Are Responsive to Drought in Soybean}

The selection of the TFRDs in Arabidopsis that are responsive to drought stress was based on data from the literature (Yamaguchi-Shinozaki and Shinozaki 2006; Shinozaki and Yamaguchi-Shinozaki 2007). The accession numbers of the ABA-dependent and ABA-independent pathway genes were obtained using the web tools of the Arabidopsis Information Resource (TAIR-http://arabidopsis.org). We use the Genevestigator website (Hruz et al. 2008) to investigate whether the pattern of expression of the AtAREB1/ABF2 (bZIP), AtMYB2, AtMYC, AtRD26/NAC, AtDREB1A/CBF3, $A t D R E B 2 A$, and $A t H D-Z I P I$ genes is upregulated or downregulated during the response to different water privation conditions or ABA stimulus (Fig. S1).

The protein sequences of the selected Arabidopsis genes and their putative paralogs were used to search all of the possible homolog TFRDs in the G. max and $O$. sativa genomes using the BLASTP tool (with the criterion of $e$ value $\leq 10^{-28}$ in the Phytozome and TAIR sites). To construct the dendrogram, we first performed a multiple alignment of the amino acid sequences for each selected gene using Clustal W2 (Larkin et al. 2007). Then, the dendrograms were constructed using 
Molecular Evolutionary Genetics Analysis (MEGA) version 4.0 (Tamura et al. 2007) by means of the neighbor-joining algorithm (Crandall et al. 2008) under a Poisson model and using the complete deletion option. Tree reconstruction was performed using the Interior Branch Test of Phylogeny and bootstrapping (1000 replications) (Sitnikova et al. 1995). The summary of this search strategy is illustrated Fig. S2.

\section{Methods, Plant Material, and Drought Stress Treatments}

We used the soybean cultivars BR 16 and Embrapa 48, which have different responses when subjected to periods of drought. The BR 16 cultivar is sensitive to water stress, while the Embrapa 48 cultivar shows a higher capacity to tolerate periods of drought (Casagrande et al. 2001; Oya et al. 2004; Texeira et al. 2008; Vidal et al. 2012). The drought assays were conducted in both a PSys in which the plants were grown in sand and a HSys in which the plants were grown in a nutrient solution (Martins et al. 2008; Kulcheski et al. 2010).

The plants that were grown under PSys conditions were maintained at a controlled temperature $\left(30 \pm 5{ }^{\circ} \mathrm{C}\right)$, $60 \pm 20 \%$ relative humidity, and a natural photoperiod. The seeds from both of the genotypes were germinated in washed sand. After approximately 10 days, the seedlings of each genotype were transplanted into $10-\mathrm{L}$ pots containing soil and bovine fertilizer in the proportion 3:1. Plants at the V4 developmental stage (third trifoliate leaf fully expanded) (Fehr and Caviness 1977) were submitted to irrigation (control) or water-deficit conditions by the suspension of irrigation for 10 days until reaching a water potential of $-3.0 \pm 0.2 \mathrm{MPa}$ (severe level stress). The water potential $(\Psi \mathrm{w})$ of each plant was measured at predawn (between 05:00 and 06:00 am) in the fourth or fifth leaf from the apex using a Scholander-type pressure chamber. The photosynthetic rate, stomatal conductance, intercellular $\mathrm{CO}_{2}$ concentration, transpiration rate, and leaf temperature during water deficit were evaluated using a LI-6400 Portable Photosynthesis System (LI-COR, Inc.). The roots were removed from the pot and immediately rinsed with water for $1 \mathrm{~min}$ by gentle agitation to remove the adhering sand. Biological contaminants were removed by careful immersion in SDS solution (2\%) for $1 \mathrm{~min}$, followed by a gentle wash in ultrapure water for $1 \mathrm{~min}$. Finally, the root samples were immediately frozen in liquid nitrogen and stored at $-80{ }^{\circ} \mathrm{C}$ for further RNA extraction. Two independent biological samples for each experimental condition were collected for the relative expression studies.

Under HSys conditions, the seeds of both of the cultivars were placed on moist filter paper and pregerminated in the dark at $25 \pm 1{ }^{\circ} \mathrm{C}$ and $65 \pm 5 \%$ relative humidity. The plantlets were transferred to polystyrene supports, and their roots were maintained completely immersed in a nutrient solution ( $\mathrm{pH}$ balanced at 6.6 and aerated) under a natural photoperiod (photosynthetic photon flux density
$($ PPFD $)=1.5 \times 10^{3} \mu \mathrm{mol} \mathrm{m} \mathrm{m}^{-2} \mathrm{~s}^{-1}$, equivalent to $8.93 \times 10^{4} \mathrm{~lx}$ ) with a 12 -h day length at $25 \pm 2{ }^{\circ} \mathrm{C}$ and $60 \pm 5 \%$ relative humidity. After 2 weeks, the seedlings at the V4 developmental stage from both of the genotypes were removed from the HSys conditions and kept in a tray in the dark without nutrient solution or water for different water privation periods: $0 \mathrm{~min}$ ( $\mathrm{T} 0$, control), $50 \mathrm{~min}$ (T50), $100 \mathrm{~min}$ (T100), and $150 \mathrm{~min}$ (T150). To typify the water deficit, the photosynthetic rate, stomata conductance, intercellular $\mathrm{CO}_{2}$ concentration, transpiration rate, and leaf temperature were evaluated using a LI-6400 Portable Photosynthesis System (LI-COR, Inc.). Measurements were taken on the fully expanded middle leaflet of the basal second leaf node under a photon flux density of $1000 \mu \mathrm{mol} \mathrm{m} \mathrm{m}^{-2} \mathrm{~s}^{-1}$. Two independent biological samples (biological replicates) for each experimental condition were collected for the relative expression studies. The root samples were harvested from a pool of ten plants. After each treatment, the samples were immediately frozen in liquid nitrogen, followed by storage at $-80{ }^{\circ} \mathrm{C}$ for posterior RNA extraction.

ABA assays were also performed to evaluate the expression of genes belonging to the classical ABA-dependent and ABA-independent pathways using the soybean cultivars BR 16 and Embrapa 48. The seeds from both of the genotypes were germinated in washed sand. After approximately 10 days, the seedlings were transplanted into $10-\mathrm{L}$ pots containing soil and bovine fertilizer in the proportion 3:1. The plants were maintained under a controlled temperature $\left(30 \pm 5{ }^{\circ} \mathrm{C}\right), 60 \pm 20 \%$ relative humidity, and a natural photoperiod. The leaves were washed with ultrapure water to remove residues of the ABA solution. The leaves were collected and submitted to different treatments: The control plants were sprayed with water, while the test plants were submitted to $300 \mathrm{ppm}$ of ABA phytohormone. Finally, after $24 \mathrm{~h}$, the treatments of the leaf samples were immediately frozen in liquid nitrogen and stored at $-80{ }^{\circ} \mathrm{C}$ for further RNA extraction. Two independent biological replicates for each condition (each composed of three plants) were used in the relative expression studies. The leaf samples from one plant from each treatment were immediately frozen in liquid nitrogen, followed by storage at $-80{ }^{\circ} \mathrm{C}$ for posterior RNA extraction.

\section{Total RNA Isolation}

The total RNA from the root samples that grew under PSys conditions was isolated using the Plant RNeasy kit (Qiagen), while the RNA from the root samples that grew under HSys conditions and from the leaf samples from the ABA experiments was isolated using the Trizol® Reagent (Invitrogen) following the manufacturer's protocol. The RNA samples were treated with RNase-free DNase I (Biolabs) in order to 
eliminate any DNA contamination and to purify the leaf samples using a cleanup kit (Qiagen). The RNA integrity was verified by electrophoresis on a $1 \%$ agarose gel, while the RNA concentration and purity were determined using a NanoDrop ${ }^{\mathrm{TM}}$ spectrophotometer ND-1000 (Thermo Scientific).

The expression of the TFRDs in both of the soybean cultivars under stress conditions under different water privation conditions or under ABA stimulus was evaluated by qPCR analysis. Primer pairs of 19 to $24 \mathrm{bp}$ and a Tm of $60 \pm 1{ }^{\circ} \mathrm{C}$ that were designed with Primer 3 plus software (Untergasser et al. 2007) were used to amplify a region of 80 to $200 \mathrm{bp}$ of the target gene. To normalize the target gene expression under water-deficit conditions, we used GmaxFbox (F-Box protein family) and GmaxUKN2 (unknown) as reference genes (RGs) (Hu et al. 2009; Kulcheski et al. 2010). To normalize target gene expression in the ABA stimulus assays, we used GmaxACT11 (cytoskeletal structural protein) and GmaxFbox as RGs (Hu et al. 2009; Kulcheski et al. 2010). The stability of each of these RGs under our conditions was determined through the NormFinder program to ensure the accuracy of the qPCR results (Andersen et al. 2004) (data not shown). The primer sequences and amplicon lengths are provided in Table S1.

Both the TFRDs and RGs from the drought stress experiments were amplified using the One-Step Kit from One-Step. The qPCR assays were carried out in a Realplex 4 Eppendorf Mastercycler Ep gradient (Eppendorf) sequence detection system, using the Power SYBR@ Green RNA-to-Ct ${ }^{\mathrm{TM}}$ One-Step Kit (Applied Biosystem) as recommended by the manufacturer's protocol. For each sample, $25 \mathrm{ng}$ of RNA was used in the reaction mixtures in a final volume of $20 \mu \mathrm{L}$. All of the samples were evaluated in technical triplicates, including a negative control. The reaction mixtures were incubated for $30 \mathrm{~min}$ at $48{ }^{\circ} \mathrm{C}$, followed by $10 \mathrm{~min}$ at $95{ }^{\circ} \mathrm{C}$ and 40 amplification cycles of $15 \mathrm{~s}$ at $95{ }^{\circ} \mathrm{C}$ and $1 \mathrm{~min}$ at $60{ }^{\circ} \mathrm{C}$ (fluorescence measurement step). At the end of 40 cycles, a melting curve was included $\left(15 \mathrm{~s}\right.$ at $95{ }^{\circ} \mathrm{C}, 15 \mathrm{~s}$ at $60{ }^{\circ} \mathrm{C}$-fluorescence measurement step-and $15 \mathrm{~s}$ at $\left.95{ }^{\circ} \mathrm{C}\right)$.

In contrast, for the ABA stimulus experiments, each single strand of complementary DNA (cDNA) was synthesized by adding $1 \mu \mathrm{g}$ of total RNA, $50 \mu \mathrm{M}$ poly-dT (oligo dT20), and $10 \mu \mathrm{M}$ each deoxyribonucleoside 5 '-triphosphate (dNTPs). This mixture was incubated at $65{ }^{\circ} \mathrm{C}$ for $5 \mathrm{~min}$ and briefly chilled on ice. Then, $20 \mathrm{mM}$ dithiothreitol (DTT), $1 \times$ firststrand buffer, and $200 \mathrm{U}$ of reverse transcriptase SuperScript ${ }^{\mathrm{TM}}$ III (Invitrogen) were added to the mixture, and the total volume $(20 \mu \mathrm{L})$ was incubated at $50{ }^{\circ} \mathrm{C}$ for $1 \mathrm{~h}$ following the manufacturer's instructions. The reverse transcriptase was inactivated by incubating the mixture at $70{ }^{\circ} \mathrm{C}$ for $15 \mathrm{~min}$, and the solution was stored at $-20{ }^{\circ} \mathrm{C}$. For each
qPCR reaction, we added $10 \mu \mathrm{L}$ of diluted cDNA (1:50), $0.2 \mu \mathrm{M}$ each primer, $50 \mu \mathrm{M}$ each dNTP, $1 \times$ PCR buffer (Invitrogen), $3 \mathrm{mM} \mathrm{MgCl}{ }_{2}, 2 \mu \mathrm{L}$ of SYBR® Green I (Molecular Probes) diluted in water (1:1000), and $0.25 \mathrm{U}$ of Platinum Taq DNA polymerase (Invitrogen) in a total volume of $20 \mu \mathrm{L}$. The reaction mixtures were incubated for $5 \mathrm{~min}$ at $94{ }^{\circ} \mathrm{C}$, followed by 40 amplification cycles of $15 \mathrm{~s}$ at $94{ }^{\circ} \mathrm{C}$, $10 \mathrm{~s}$ at $60{ }^{\circ} \mathrm{C}$ (fluorescence measurement step), and $15 \mathrm{~s}$ at $72{ }^{\circ} \mathrm{C}$. At the end of 40 cycles, a melting curve was included (15 s at $95{ }^{\circ} \mathrm{C}, 15 \mathrm{~s}$ at $60{ }^{\circ} \mathrm{C}$ - fluorescence measurement step-and 15 s at $95^{\circ} \mathrm{C}$ ).

Polymerase chain reactions were performed in the 7500 Fast Real-Time PCR detection system (Applied Biosystem) using SYBR ${ }^{\circledR}$ Green to monitor cDNA synthesis. The PCR efficiencies and optimal quantification cycle threshold or $\mathrm{Cq}$ value were estimated using the Real-Time PCR Miner software (Zhao and Fernald 2005). Two independent biological samples of each experimental condition were evaluated using technical replicates. The relative expression level of the drought stress sample from the water-deficit experiments was calculated using the relative expression software tool (RESTC), which compares two treatment groups with multiple data points in the sample versus control groups and calculates the relative expression ratio between them. The mathematical model that was used is based on the PCR efficiencies and the mean $\mathrm{Cp}$ deviation of target genes between the sample and the control group, normalized by the mean $\mathrm{Cp}$ deviation of the reference genes (Pfaffl et al. 2002). The advantage REST is the provision of a subsequent statistical test of the analyzed Cp values by a Pair-Wise Fixed Reallocation Randomization Test (Pfaffl et al. 2002). The relative expression level of the ABA experiments was calculated using the values of the cutting cycle (quantification cycle value- $\mathrm{Cp}$ ), which were converted in the program qBase version 1.3.5 (Hellemans et al. 2007). A statistical analysis was performed using Student's $t$ test.

\section{Promoter Analysis}

The promoter sequences of the TFRDs (1000 bp upstream of the start codon) were obtained from the genome browser tool in the Phytozome database. Cis-regulatory elements that were responsive to drought stress, salinity stress, osmotic stress, and ABA (Table S2) were identified through web tool in the database of plant cis program-acting regulatory DNA elements (PLACE; Higo et al. 1999) and from previous studies (Nakashima et al. 2006; Umezawa et al. 2010). The frequency of the selected Cis-regulatory elements in the promoter region of each TFRD was compared to the expected frequency in the genes of the G. max genome. The statistical analyses of the $\mathrm{Cis}$ elements of the TFRD promoters were performed using the POBO web tool (Kankainen and Holm 2004). 
Acknowledgments We thank Durvalina Felix for the technical support. This work was part of Guimarães-Dias's $\mathrm{PhD}$ research in Genetics, at the Department of Genetics of the Universidade Federal do Rio de Janeiro (UFRJ), and was supported by the Conselho Nacional de Desenvolvimento Científico e Tecnológico (CNPq; M. Alves-Ferreira) and the Fundação de Amparo à Pesquisa do Rio de Janeiro (FAPERJ; M. Alves-Ferreira). The graduate $\mathrm{PhD}$ scholarship of F. Guimarães-Dias was granted by CNPq.

Authors' Contributions MA-F, ACNB, and FGD planned and supervised the study. MA-F, ACNB, and FGD contributed to the design and execution of the experiments and drafted the manuscript. LGK and AJC performed the expression analysis in the ABA samples. RMSA, MELS, AVJF, FGD, MAB, and ER contributed to the RNA extraction and expression analysis in the drought stress samples. ROM and MEL contributed to the development of the PSys experiment. FAR and AN contributed to the development of the HSys experiment. MAF, ACNB, and FGD contributed to the interpretation of the data and provided intellectual input. AN and MFGDS revised the manuscript and financial support. All of the authors have read and approved the final manuscript.

\section{Compliance with Ethical Standards}

Competing Interests The authors declare that they have no competing interests.

\section{References}

Abe H, Urao T, Ito T, Seki M, Shinozaki K, Yamaguchi-Shinozaki K (2003) Arabidopsis AtMYC2 (bHLH) and AtMYB2 (MYB) function as transcriptional activators in Abscisic acid signaling. Plant Cell Online 15:63-78

Andersen C, Jensen J, Orntoft T (2004) Normalization of real-time quantitative reverse transcription-PCR data: a model-based variance estimation approach to identify genes suited for normalization, applied to bladder and colon cancer data sets. Cancer Res 64(15):5245-5250

Aubert Y, Leba L-J, Cheval C, Ranty B, Vavasseur A, Aldon D, Galaud JP (2011) Involvement of RD20, a member of caleosin family, in ABA-mediated regulation of germination in Arabidopsis thaliana. Plant Signal Behav 6:538-540

Avramova Z (2015) Transcriptional 'memory' of a stress: transient chromatin and memory (epigenetic) marks at stress-response genes. Plant J 83:149-159

$\mathrm{Bu} \mathrm{Q}$ et al. (2008) Role of the Arabidopsis thaliana NAC transcription factors ANAC019 and ANAC055 in regulating jasmonic acidsignaled defense responses. Cell Res 18:756-767

Busk PK, Pagès M (1998) Regulation of abscisic acid-induced transcription. Plant Mol Biol 37:425-435

Casagrande EC, et al. (2001) Expressão gênica diferencial durante déficit hídrico em soja. Rev Bras Fisiol Veg 13:168-184

Clemente TE, Cahoon EB (2009) Soybean oil: genetic approaches for modification of functionality and total content. Plant Physiol 151: 1030-1040. doi:10.1104/pp.109.146282

Comlekcioglu N, Simsek M (2011) Effects of deficit irrigation on yield and yield components of vegetable soybean Glycine max L. (Merr.) in semi-arid conditions. Afr J Biotechnol 10:6227-6234

Cowan IR (1965) Transport of water in the soil-plant-atmosphere system. J Appl Ecol 2:221-239
Crandall K, Lagergren J, Simonsen M, Mailund T, Pedersen C (2008) Rapid neighbour-joining. In: Algorithms in bioinformatics, vol 5251. Lecture notes in computer science. Springer Berlin/ Heidelberg, pp 113-122. doi:10.1007/978-3-540-87361-7_10

de Paiva Rolla AA et al. (2014) Phenotyping soybean plants transformed with rd29A:AtDREB1A for drought tolerance in the greenhouse and field Transgenic. Research 23:75-87. doi:10.1007/s11248013-9723-6

Ding Y, Fromm M, Avramova Z (2012) Multiple exposures to drought 'train' transcriptional responses in Arabidopsis. Nat Commun 3 doi:10.1038/ncomms 1732

Ding Y, Liu N, Virlouvet L, Riethoven JJ, Fromm M, Avramova Z (2013) Four distinct types of dehydration stress memory genes in Arabidopsis thaliana. BMC Plant Biol 13:1-11. doi:10.1186/14712229-13-229

Doyle JJ, Egan AN (2010) Dating the origins of polyploidy events. New Phytol 186:73-85. doi:10.1111/j.1469-8137.2009.03118.x

Fan CM, Wang X, Wang YW, Hu RB, Zhang XM, Chen JX, Fu YF (2013) Genome-wide expression analysis of soybean MADS genes showing potential function in the seed development. PLoS ONE 8: e62288. doi:10.1371/journal.pone.0062288

Fang Y, Xiong L (2015) General mechanisms of drought response and their application in drought resistance improvement in plants. Cell Mol Life Sci 72(4):673-689

Fehr W, Caviness C (1977) Stages of soybean development. Iowa State University, Ames, IA

Ferreira Neto JRC, Pandolfi V, Guimaraes FCM, Benko-Iseppon AM, Romero C, Silva RLDO, Rodrigues FA, Abdelnoor RV, Nepomuceno AL, Kido EA (2013) Early transcriptional response of soybean contrasting accessions to root dehydration. PLoS One 8(12):e83466

Fujimoto R, Sasaki T, Ishikawa R, Osabe K, Kawanabe T, Dennis ES (2012) Molecular mechanisms of epigenetic variation in plants. Int J Mol Sci 13(8):9900-9922

Fujita M et al. (2004) A dehydration-induced NAC protein, RD26, is involved in a novel ABA-dependent stress-signaling pathway. Plant J 39:863-876

Guimarães-Dias F, Neves-Borges AC, Viana AAB, Mesquita RO, Romano E, Grossi-de-Sá MF, Nepomuceno AL, Loureiros ME, Alves-Ferreira M (2012) Expression analysis in response to drought stress in soybean: shedding light on the regulation of metabolic pathway genes. Genet Mol Biol 35(1):222-232

Harb A, Krishnan A, Ambavaram MMR, Pereira A (2010) Molecular and physiological analysis of drought stress in Arabidopsis reveals early responses leading to acclimation in plant growth. Plant Physiol 154(3):1254-1271. doi:10.1104/pp.110.161752

Hellemans J, Mortier G, De Paepe A, Speleman F, Vandesompele J (2007) qBase relative quantification framework and software for management and automated analysis of real-time quantitative PCR data. Genome Biol 8:R19

Higo K, Ugawa Y, Iwamoto M, Korenaga T (1999) Plant cis-acting regulatory DNA elements (PLACE) database: 1999. Nucleic Acids Res 27(1):297-300

Hoque TS, Hossain MA, Mostofa MG, Burritt DJ, Fujita M (2016) Signalling roles of methylglyoxal and the involvement of the glyoxalase system in plant abiotic stress responses and tolerance. In: Plant-Environment Interaction. John Wiley \& Sons, Ltd, pp 311-326

Hruz T, Laule O, Szabo G, Wessendorp F, Bleuler S, Oertle L, Widmayer P, Gruissem W, Zimmermann P (2008) Genevestigator V3: a reference expression database for the meta-analysis of transcriptomes. Adv Bioinforma 2008

Hu R, Fan C, Li H, Zhang Q, Fu Y-F (2009) Evaluation of putative reference genes for gene expression normalization in soybean by quantitative real-time RT-PCR. BMC Mol Biol 10(1):93 
Hussain SS, Kayani MA, Amjad M (2011) Transcription factors as tools to engineer enhanced drought stress tolerance in plants. Biotechnol Prog 27(2):297-306. doi:10.1002/btpr.514

Kankainen M, Holm L (2004) POBO, transcription factor binding site verification with bootstrapping. Nucleic Acids Res 32:W222-W229

Kazan K, Manners JM (2013) MYC2: the master in action. Mol Plant 6: 686-703

Kilian J, Peschke F, Berendzen KW, Harter K, Wanke D (2012) Prerequisites, performance and profits of transcriptional profiling the abiotic stress response. Biochim Biophys Acta Gene Regul Mech 1819(2):166-175. doi:10.1016/j.bbagrm.2011.09.005

Kim J-M, Sasaki T, Ueda M, Sako K, Seki M (2015) Chromatin changes in response to drought, salinity, heat, and cold stresses in plants. Front Plant Sci 6:114. doi:10.3389/fpls.2015.00114

Kulcheski FR, Marcelino-Guimaraes FC, Nepomuceno AL, Abdelnoor RV, Margis R (2010) The use of microRNAs as reference genes for quantitative polymerase chain reaction in soybean. Anal Biochem 406(2): 185-192

Larkin MA, Blackshields G, Brown NP, Chenna R, McGettigan PA, McWilliam H, Valentin F, Wallace IM, Wilm A, Lopez R, Thompson JD, Gibson TJ, Higgins DG (2007) Clustal W and clustal $X$ version 2.0. Bioinformatics 23:2947-2948

Le DT, Nishiyama R, Watanabe Y, Mochida K, Yamaguchi-Shinozaki K, Shinozaki K, Tran L-SP (2011) Genome-wide survey and expression analysis of the plant-specific NAC transcription factor family in soybean during development and dehydration stress. DNA Res. doi:10.1093/dnares/dsr015

Lorenzo O, Chico JM, Sánchez-Serrano JJ, Solano R (2004) JASMONATE-INSENSITIVE1 encodes a MYC transcription factor essential to discriminate between different Jasmonate-regulated defense responses in Arabidopsis. Plant Cell 16:1938-1950

Lynch J (1995) Root architecture and plant productivity. Plant Physiol 109(1):7-13. doi:10.1104/pp.109.1.7

Marcolino-Gomes J, Rodrigues FA, Neves Oliveira MC, Boucas Farias JR, Neumaier N, Abdelnoor RV, Marcelino-Guimaraes FC, Nepomuceno AL (2013) Expression patterns of GmAP2/EREBlike transcription factors involved in soybean responses to water deficit. PLoS One 8(5):e62294. doi:10.1371/journal.pone.0062294

Martins PK, Jordao BQ, Yamanaka N, Farias JRB, Beneventi MA, Binneck E, Fuganti R, Stolf R, Nepomuceno AL (2008) Differential gene expression and mitotic cell analysis of the drought tolerant soybean (Glycine max L. Merrill Fabales, Fabaceae) cultivar MG/BR46 (Conquista) under two water deficit induction systems. Genet Mol Biol 31(2):512-521

Mayer Weber RL, Wiebke-Strohm B, Bredemeier C, Margis-Pinheiro M, de Brito GG, Rechenmacher C, Bertagnolli PF, Lisei de Sa ME, Campos MdA, Santos de Amorim RM, Beneventi MA, Margis R, Grossi-de-Sa MF, Bodanese-Zanettini MH (2014) Expression of an osmotin-like protein from Solanum nigrum confers drought tolerance in transgenic soybean. Bmc Plant Biol 14. doi:10.1186/ s12870-014-0343-y

Miyamoto K, Nishizawa Y, Minami E, Nojiri H, Yamane H, Okada K (2015) Overexpression of the bZIP transcription factor OsbZIP79 suppresses the production of diterpenoid phytoalexin in rice cells. J Plant Physiol 173:19-27. doi:10.1016/j.jplph.2014.09.001

Munns R, James RA, Sirault XRR, Furbank RT, Jones HG (2010) New phenotyping methods for screening wheat and barley for beneficial responses to water deficit. J Exp Bot 61(13):3499-3507

Mutava RN, Prince SJK, Syed NH, Song L, Valliyodan B, Chen W, Nguyen HT (2015) Understanding abiotic stress tolerance mechanisms in soybean: a comparative evaluation of soybean response to drought and flooding stress. Plant Physiol Biochem 86:109-120. doi:10.1016/j.plaphy.2014.11.010

Nakashima K, Fujita Y, Katsura K, Maruyama K, Narusaka Y, Seki M, Shinozaki K, Yamaguchi-Shinozaki K (2006) Transcriptional regulation of ABI3-and ABA-responsive genes including RD29B and
RD29A in seeds, germinating embryos, and seedlings of Arabidopsis. Plant Mol Biol 60(1):51-68

Nakashima K, Yamaguchi-Shinozaki K, Shinozaki K (2014) The transcriptional regulatory network in the drought response and its crosstalk in abiotic stress responses including drought, cold, and heat. Front Plant Sci 5:170. doi:10.3389/fpls.2014.00170

Narusaka Y et al. (2003) Interaction between two cis-acting elements, ABRE and DRE, in ABA-dependent expression of Arabidopsis rd29A gene in response to dehydration and high-salinity stresses the. Plant J 34:137-148

Neves-Borges AC et al. (2012) Expression pattern of drought stress marker genes in soybean roots under two water deficit systems. Genet Mol Biol 35:212-221

Osakabe Y, Osakabe K, Shinozaki K, Tran L-SP (2014) Response of plants to water stress. Front Plant Sci 5:86. doi:10.3389 /fpls.2014.00086

Oya T, Nepomuceno AL, Neumaier N, Farias JRB, Tobita S, Ito O (2004) Drought tolerance characteristics of Brazilian soybean cultivarsevaluation and characterization of drought tolerance of various Brazilian soybean cultivars in the field. Plant Prot Sci 7(2):129 137. doi:10.1626/pps.7.129

Pandey A, Misra P, Bhambhani S, Bhatia C, Trivedi PK (2014) Expression of Arabidopsis MYB transcription factor, AtMYB111, in tobacco requires light to modulate flavonol content. Sci Report 4: 5018. doi:10.1038/srep05018

Pfaffl MW, Horgan GW, Dempfle L (2002) Relative expression software tool (REST (c)) for group-wise comparison and statistical analysis of relative expression results in real-time PCR. Nucleic Acids Res 30(9):e36. doi:10.1093/nar/30.9.e36

Qiao Z, Li C-L, Zhang W (2016) WRKY1 regulates stomatal movement in drought-stressed Arabidopsis thaliana Plant Mol Biol:1-13

Sahu P, Pandey G, Sharma N, Puranik S, Muthamilarasan M, Prasad M (2013) Epigenetic mechanisms of plant stress responses and adaptation. Plant Cell Rep 32(8):1151-1159. doi:10.1007/s00299-013$1462-x$

Savitri E, Basuki N, Aini N, Arumingtyas E (2013) Identification and characterization drought tolerance of gene LEA-D11 soybean (Glycine max L. Merr) based on PCR-sequencing. Am J Mol Biol 3:32-37. doi:10.4236/ajmb.2013.31004

Schmutz J, Cannon SB, Schlueter J, Ma JX, Mitros T, Nelson W, Hyten DL, Song QJ, Thelen JJ, Cheng JL, Xu D, Hellsten U, May GD, Yu Y, Sakurai T, Umezawa T, Bhattacharyya MK, Sandhu D, Valliyodan B, Lindquist E, Peto M, Grant D, Shu SQ, Goodstein D, Barry K, Futrell-Griggs M, Abernathy B, Du JC, Tian ZX, Zhu LC, Gill N, Joshi T, Libault M, Sethuraman A, Zhang XC, Shinozaki K, Nguyen HT, Wing RA, Cregan P, Specht J, Grimwood J, Rokhsar D, Stacey G, Shoemaker RC, Jackson SA (2010) Genome sequence of the palaeopolyploid soybean. Nature 463(7278):178-183

Shinozaki K, Yamaguchi-Shinozaki K (1996) Molecular responses to drought and cold stress. Curr Opin Biotechnol 7:161-167

Shinozaki K, Yamaguchi-Shinozaki K (2007) Gene networks involved in drought stress response and tolerance. J Exp Bot 58(2):221-227. doi:10.1093/jxb/erl164

Sitnikova T, Rzhetsky A, Nei M (1995) Interior-branched and bootstrap tests of phylogenetic trees. Mol Biol Evol 12(2):319-333

Stolf-Moreira R, Medri M, Neumaier N, Lemos N, Pimenta J, Tobita S, Brogin R, Marcelino-Guimarães F, Oliveira M, Farias J, Abdelnoor R, Nepomuceno A (2010b) Soybean physiology and gene expression during drought. Genet Mol Res 5:1946-1956

Stolf-Moreira R, Lemos E, Carareto-Alves L, Marcondes J, Pereira S, Rolla A, Pereira R, Neumaier N, Binneck E, Abdelnoor R, de Oliveira M, Marcelino F, Farias J, Nepomuceno A (2011) Transcriptional profiles of roots of different soybean genotypes subjected to drought stress. Plant Mol Biol Report 29(1):19-34. doi:10.1007/s11105-010-0203-3 
Tamura K, Dudley J, Nei M, Kumar S (2007) MEGA4: molecular evolutionary genetics analysis (MEGA) software version 4.0. Mol Biol Evol 24(8):1596-1599

Texeira LR, de Lucca E, Braccini A, Sperandio D, Scapim CA, Schuster I, Vigano J (2008) Evaluation of soybean cultivars regarding tolerance to water stress in substrat containing polyethylene glycol. Acta Sci Agron 30(2):217-223

Thao NP, Tran L-SP (2012) Potentials toward genetic engineering of drought-tolerant soybean. Crit Rev Biotechnol 32(4):349-362. doi: $10.3109 / 07388551.2011 .643463$

Tran L-S, Mochida K (2010) Functional genomics of soybean for improvement of productivity in adverse conditions. Funct Integr Genomics 10(4):447-462. doi:10.1007/s10142-010-0178-z

Umezawa T, Nakashima K, Miyakawa T, Kuromori T, Tanokura M, Shinozaki K, Yamaguchi-Shinozaki K (2010) Molecular basis of the core regulatory network in ABA responses: sensing, signaling and transport. Plant Cell Physiol 51(11):1821-1839. doi:10.1093 /pcp/pcq156

Untergasser A, Nijveen H, Rao X, Bisseling T, Geurts R, Leunissen JAM (2007) Primer3Plus, an enhanced web interface to Primer3. Nucleic Acids Res 35:W71-W74

Vadez V (2014) Root hydraulics: the forgotten side of roots in drought adaptation. Field Crop Res 165:15-24. doi:10.1016/j.fcr.2014.03.017

Vidal RO, do Nascimento LC, Mondego JMC, Pereira GAG, Carazzolle MF (2012) Identification of SNPs in RNA-seq data of two cultivars of Glycine max (soybean) differing in drought resistance. Genet Mol Biol 35(1 Suppl):331-334. doi:10.1590/s 141547572012000200014

Wang $\mathrm{H}$ et al. (2012) Expression of an apoplast-localized BURP-domain protein from soybean (GmRD22) enhances tolerance towards abiotic stress plant. Cell Environ 35:1932-1947

Xu Z-S, Chen M, Li L-C, Ma Y-Z (2011) Functions and application of the AP2/ERF transcription factor family in crop improvement. J Integr Plant Biol 53(7):570-585. doi:10.1111/j.1744-7909.2011.01062.x

Yamaguchi-Shinozaki K, Shinozaki K (1993) The plant hormone abscisic acid mediates the drought-induced expression but not the seedspecific expression of $\mathrm{rd} 22$, a gene responsive to dehydration stress in Arabidopsis thaliana. Mol Gen Genet MGG 238:17-25. doi:10.1007/bf00279525

Yamaguchi-Shinozaki K, Shinozaki K (2006) Transcriptional regulatory networks in cellular responses and tolerance to dehydration and cold stresses. Annu Rev Plant Biol 57:781-803

Yoshida T, Mogami J, Yamaguchi-Shinozaki K (2014) ABA-dependent and $\mathrm{ABA}$-independent signaling in response to osmotic stress in plants. Curr Opin Plant Biol 21:133-139. doi:10.1016/j. pbi.2014.07.009

Zhao S, Fernald RD (2005) Comprehensive algorithm for quantitative real-time polymerase chain reaction. J Comput Biol 12(8):10471064. doi: $10.1089 / \mathrm{cmb} .2005 .12 .1047$ 